\title{
ON THE FREQUENCY OF TITCHMARSH'S PHENOMENON FOR $\zeta(s)$-VII
}

\section{K. Ramachandra}

\section{Introduction}

In this paper we study small intervals $I$ for $t$ (of length $H \geq \exp \exp (e)$ ) contained in $[T, 2 T]$ for which

$$
\max _{t \in I}|\zeta(1+i t)| \geq e^{\gamma}(\log \log H-\log \log \log H-\varrho),
$$

where $\gamma$ is the Euler's constant and $\varrho$ a certain real constant which is effective. All our constants including the 0 -constants are effective. The Greek letter $\theta$ will denote the least upper bound of the real parts of the zeros of $\zeta(s)$. In the present state of knowledge we do not know whether $\theta<1$ or not. Only one of our results depends on the hypothesis $\theta<1$ (in place of the more drastic Riemann hypothesis which asserts that $\theta=1 / 2$ ) and in this case it is only for convenience that we assume that $\theta$ is effective. The $n^{\text {th }}$ iterated $\operatorname{logarithm} \log _{n} T$ is defined inductively $\log _{1} T=\log T$, and $\log _{n+1} T=\log \left(\log _{n} T\right)$. Similarly the $n^{\text {th }}$ iterated exponential is defined by $\exp _{1}(T)=\exp (T)$ and $\exp _{n+1}(T)=\exp \left(\exp _{n}(T)\right)$. Our first result is that the inequality (1) holds for all $H$ satisfying

$$
T \geq H \geq C_{1} \log _{4} T
$$

where $C_{1} \geq 1$ is a certain constant. Our next result is that if the hypothesis $\theta<1$ is true, then (1) holds for all $H$ satisfying

$$
T \geq H \geq C_{2} \log _{5} T
$$

where $C_{2} \geq 1$ is a certain constant. We assume throughout that $T \geq C_{3}$ and $H \geq C_{4}$ where $C_{3}$ and $C_{4}$ are certain positive constants. Let now $H<C_{1} \log _{4} T$. Consider a set of disjoint intervals $I$ contained in $[T, 2 T]$ for which (1) is false. Our third result asserts that the number of such disjoint intervals does not exceed $T X_{1}^{-1}$ where $X_{1}=\exp _{4}(\beta H)$, where $\beta>0$ is a constant. Again let $H<$ $C_{2} \log _{5} T$. Consider a set of disjoint intervals $I$ contained in $[T, 2 T]$ for which (1) is false. Our (fourth and) final result asserts that the number of such disjoint intervals does not exceed $T X_{2}^{-1}$ where $X_{2}=\exp _{5}\left(\beta^{\prime} H\right)$ where $\beta^{\prime}>0$ is a constant. Similar results can be proved for $|\zeta(1+i t)|^{-1}$. We have only to replace $e^{\gamma}$ by $6 e^{\gamma} / \pi^{2}$. These results can be generalized suitably to $\zeta$ and $L$-functions of algebraic number fields and so on. 


\section{Titchmarsh series and a main theorem}

The study referred to in the introduction is based (apart from other ideas) on the following Theorem B due essentially to the author [7] (see also [8] and [3]). There the author proved the following two Theorems A and B. We begin with the following definition.

Titchmarsh series. Let $A \geq 1$ be a constant. Let $1=\lambda_{1}<\lambda_{2}<\lambda_{3}<\cdots$ where $1 / A \leq \lambda_{n+1}-\lambda_{n} \leq A$. Let $1=a_{1}, a_{2}, a_{3}, \ldots$ be a sequence of complex numbers, possibly depending on a parameter $H(\geq 10)$ such that $\left|a_{n}\right| \leq\left(\lambda_{n} H\right)^{A}$. Put $F(s)=\sum_{n=1}^{\infty} a_{n} \lambda_{n}^{-s}$ where $s=\sigma+i t$. Then $F(s)$ is analytic in $\sigma \geq A+2$. $F(s)$ is called a Titchmarsh series if there exists a constant $A \geq 1$ with the above properties and further a system of infinite rectangles $R(T, T+H)$ defined by $\{\sigma \geq 0, T \leq t \leq T+H\}$ where $10 \leq H \leq T$ and $T$ (which may be related to $H$ ) tends to infinity and $F(s)$ admits an analytic continuation into these rectangles and the maximum of $|F(s)|$ taken over $R(T, T+H)$ does not exceed $\exp _{2}(H / 80 A)$.

Remark. It suffices for all our purposes to assume that $|F(s)|$ is continuous in $R(T, T+H)$ and that $F(s)$ is analytic in $\{\sigma>0, T \leq t \leq T+H\}$ besides the other properties.

Theorem A. We have

$$
\frac{1}{H} \int_{L}|F(i t)| d t>C_{A}
$$

where $C_{A}>0$ depends only on $A$ and $L$ is the side $\{\sigma=0, T \leq t \leq T+H\}$ of $R(T, T+H)$.

Theorem B. We have

$$
\frac{1}{H} \int_{L}|F(i t)|^{2} d t>C_{A} \sum_{\lambda_{n} \leq X}\left|a_{n}\right|^{2}\left(1-\frac{\log \lambda_{n}}{\log H}+\frac{1}{\log _{2} H}\right),
$$

where $X=2+D_{A} H$, and $C_{A}>0, D_{A}>0$ depend only on $A$.

Remark. If $\lambda_{n}=n$ then it was shown in [3] that $X$ can be taken to be $H / 200$. The essential point in that paper was that the tapering factor multiplying $\left|a_{n}\right|^{2}$ was improved. The bound on $|F(s)|$ was relaxed to $\exp _{2}(H / 80 A)$. (This was known to the author for quite some time.) However, for our applications Theorem B is enough and the improvement in the tapering factor does not seem to have any extra advantage for the purposes of the present paper.

From Theorem B we deduce (in the rest of this section) our main theorem.

Main Theorem. Let $I$ be an interval contained in $[T, 2 T]$ and of length $H$ and let the maximum of $|\zeta(\sigma+i t)|$ taken over the rectangle $\{\sigma \geq 1, t \in I\}$ not exceed $\exp _{2}(H / 100)$. Then

$$
\max _{t \in I}|\zeta(1+i t)| \geq e^{\gamma}\left(\log _{2} H-\log _{3} H-\varrho\right),
$$

where $\gamma$ is Euler's constant and $\varrho$ is a certain real constant. 
We do this in a series of lemmas. The deduction can be done in a somewhat similar fashion as in [2] or [10] although we follow the latter. First of all we take $F(s)=(\zeta(1+s))^{k}$ and set $k$ equal to the greatest integer not exceeding $\log H / 5 \log _{2} H$. We verify that $F(s)$ is a Titchmarsh series with $\lambda_{n}=n$ and. $a_{1}=1$. Now

$$
a_{n}=\frac{d_{k}(n)}{n}<n \sum_{m=1}^{\infty} \frac{d_{k}(m)}{m^{2}}=n(\zeta(2))^{k}<n H
$$

since $k<\log H$ and $\zeta(2)<e$. Under the conditions of the main theorem, the maximum of $|F(s)|$ in the relevant rectangle does not exceed $\exp \{(\log H) \exp (H / 100)\}$ $\leq \exp _{2}(H / 80)$, provided that $\log H \leq \exp (H / 400)$ which is certainly true if $(H / 400)^{2} \geq 2 H$, i.e., if $H \geq 320000$. Hence we can take $A=1$. Thus we have the following

Lemma 1. Under the hypothesis of the main theorem, with $k$ chosen as the greatest integer not exceeding $\log H / 5 \log _{2} H$, we have

$$
\frac{1}{H} \int_{t \in I}|\zeta(1+i t)|^{2 k} d t>C_{5} \sum_{n \leq H / 200}\left(\frac{d_{k}(n)}{n}\right)^{2}\left(1-\frac{\log n}{\log H}+\frac{1}{\log _{2} H}\right)
$$

and so

$$
\max _{t \in I}|\zeta(1+i t)| \geq\left(\frac{C_{5}}{\log _{2} H}\right)^{1 / 2 k} Q
$$

where $Q=\max _{n \leq H / 200}\left(d_{k}(n) / n\right)^{1 / k}$ and $C_{5}>0$ is a constant.

Lemma 2. We have

$$
\left(\frac{C_{5}}{\log _{2} H}\right)^{1 / 2 k}=1+O\left(\frac{\log _{2} k}{k}\right) .
$$

Proof. The lemma follows from the definition of $k$.

Lemma 3. The quantity $d_{k}(n) / n$, which is defined on prime powers by

$$
\frac{d_{k}(1)}{1}=1 \text { and } \frac{d_{k}\left(p^{m}\right)}{p^{m}}=\frac{k(k+1) \cdots(k+m-1)}{m ! p^{m}} .
$$

is a multiplicative function of $n$.

Proof. The lemma follows from the definition of $d_{k}(n)$ as the coefficient of $n^{-s}$ in $(\zeta(s))^{k}$ and the Euler product for $\zeta(s)$. 
Lemma 4. For $m \geq 0$ we have

$$
\frac{d_{k}\left(p^{m+1}\right)}{p^{m+1}}=\left(\frac{d_{k}\left(p^{m}\right)}{p^{m}}\right)\left(\frac{k+m}{(m+1) p}\right)<\frac{3}{4}\left(\frac{d_{k}\left(p^{m}\right)}{p^{m}}\right)
$$

provided $4 k+4 m<3 m p+3 p$, i.e., $m>(4 k-3 p) /(3 p-4)$.

Proof. This follows directly from Lemma 3.

Lemma 5. The inequality (6) holds if $p \leq k$ and $m \geq\left[m_{0}+1\right]$, where $m_{0}=(4 k-3 p) /(3 p-4)$. We also have

$$
m_{0}+1<\frac{4 k}{p}
$$

Proof. We have

$$
m_{0}+1=\frac{4 k-4}{3 p-4}=\frac{4 k-4}{p+2 p-4} \leq \frac{4 k-4}{p}<\frac{4 k}{p}
$$

and hence the lemma follows.

Lemma 6. We have

$$
\left(1-\frac{1}{p}\right)^{-k}<\left(m_{0}+5\right) \max _{m \leq m_{0}+1}\left(\frac{d_{k}\left(p^{m}\right)}{p^{m}}\right) .
$$

Proof. Let $\nu=\left[m_{0}+1\right]$. Then the LHS equals

$$
\sum_{m=0}^{\nu} \frac{d_{k}\left(p^{m}\right)}{p^{m}}+\sum_{m=\nu+1}^{\infty} \frac{d_{k}\left(p^{m}\right)}{p^{m}} .
$$

Here the first sum does not exceed $\left(m_{0}+2\right)$ times the maximum in question. The second sum is by (6) less than $\left((3 / 4)+(3 / 4)^{2}+(3 / 4)^{3}+\cdots\right)$ times the maximum in question. This proves the lemma.

Lemma 7. Let $p \leq k$. If $m$ denotes the integer (to avoid a complicated notation) not exceeding $m_{0}+1$ for which the maximum of $\left(d_{k}\left(p^{m}\right)\right) p^{-m}$ is attained, we have

$$
\frac{d_{k}\left(p^{m}\right)}{p^{m}} \geq \frac{p}{8 k}\left(1-\frac{1}{p}\right)^{-k}
$$

Proof. This lemma follows from (7) and (8) since $4+(4 k / p) \leq(8 k) / p$. 
Lemma 8. With $k$ as in Lemma 1, we have, for $H \geq H_{0}$,

$$
\prod_{p \leq k} p^{m} \leq \frac{H}{200}
$$

Proof. Since $m \leq m_{0}+1 \leq 4 k / p$ it suffices to check that

$$
\prod_{p \leq k} p^{4 k / p} \leq \frac{H}{200}, \quad \text { i.e., } \quad \sum_{p \leq k} \frac{\log p}{p} \leq \log H-\log (200) .
$$

The last statement follows since (by prime number theorem) $\sum_{p \leq k} \log p / p$ is asymptotic to $\log k$ as $k$ tends to infinity.

Lemma 9. We have, for $Q$ defined in Lemma 1, the lower bound given by

$$
Q \geq e^{\gamma}(\log k+O(1))
$$

Proof. By (9) it suffices to check that

$$
\left(\prod_{p \leq k}\left(\frac{p}{8 k}\right)^{1 / k}\right) \prod_{p \leq k}\left(1-\frac{1}{p}\right)^{-1} \geq e^{\gamma}(\log k+O(1)) .
$$

It is well-known that the second product in (11) is $\geq e^{\gamma}(\log k+O(1))$ (see (3.15.2) of [11] for a weaker result which is not hard to improve; see also p. 81 of Prachar's Primzahlverteilung, Springer-Verlag, 1959). The logarithm of the first product is

$$
\frac{1}{k} \sum_{p \leq k}(\log p-\log k-\log 8)=O\left(\frac{1}{\log k}\right)
$$

on using the prime number theorem in the forms

$$
\sum_{p \leq k} \log p=k+O\left(\frac{k}{\log k}\right) \quad \text { and } \quad \sum_{p \leq k} \log k=k+O\left(\frac{k}{\log k}\right) .
$$

Hence (11) follows. This completes the proof of Lemma 9.

Lemmas 2 and 9 complete the proof of the main theorem. 


\section{First application of the main theorem}

Theorem 1. Let $I$ be an interval of length $H$ contained in $[T, 2 T]$. Let $T \geq H \geq C_{1} \log _{4} T$. Then

$$
\max _{t \in I}|\zeta(1+i t)| \geq e^{\gamma}\left(\log _{2} H-\log _{3} H-\varrho\right),
$$

where $\gamma$ is Euler's constant and $\varrho$ is a certain real constant.

We prove this by a series of lemmas.

Lemma 1. Divide $I$ into three equal parts each of length $H / 3$. Denote the middle interval by $I_{2}$ and the others by $I_{1}$ and $I_{3}$. Then we have either

$$
\max _{t \in I_{2}}|\zeta(1+i t)| \geq e^{\gamma}\left(\log _{2} H-\log _{3} H-O(1)\right),
$$

or

$$
\max _{\sigma>1, t \in I_{2}}|\zeta(\sigma+i t)| \geq \exp _{2}(H / 300) .
$$

Proof. The lemma follows from the main theorem.

Lemma 2. If the maximum in (13) occurs for $1<\sigma \leq 1+\delta$, where $\delta=\left(\exp _{3}(H / 400)\right)^{-1}$, then (12) holds.

Proof. In this case we have for some $t_{0}$ in $I_{2}$ the inequality

$$
\begin{aligned}
\left|\zeta\left(1+i t_{0}\right)\right| & \geq\left|\zeta\left(\sigma+i t_{0}\right)\right|-\delta \max _{1 \leq u \leq 1+\delta}\left|\zeta^{\prime}\left(u+i t_{0}\right)\right| \\
& \geq \exp _{2}(H / 300)-\delta\left(C_{6} \log ^{2} t_{0}\right) \geq \frac{1}{2} \exp _{2}(H / 300),
\end{aligned}
$$

provided $\exp _{3}(H / 400) \geq C_{6}\left(\log t_{0}\right)^{2}$. Hence the lemma is proved.

Lemma 3. For any complex number $z$ with $|\operatorname{Re}(z)| \leq 1 / 4$, we have the inequality

$$
\left|\exp \left((\sin z)^{2}\right)\right|<<\exp (-\exp |\operatorname{Im}(z)|)
$$

where the constant implied by the Vinogradov symbol $<<$ is absolute.

Proof. Let $z=x+i y$ where $x$ and $y$ are real and $i=\sqrt{-1}$. Now

$$
\left.\operatorname{Re}(\sin z)^{2}\right)=\operatorname{Re}\left(\frac{1}{2}\left(1-\frac{1}{2}\left(e^{2 i z}+e^{-2 i z}\right)\right)\right)=\frac{1}{4}\left(2-e^{-2 y} \cos (2 x)-e^{2 y} \cos (2 x)\right) .
$$

Note that in $|x| \leq 1 / 4, \cos (2 x)$ is positive and is greater than or equal to $\cos (1 / 2) \geq \cos (\pi / 6=\sqrt{3} / 2$. Hence

$$
\left.\operatorname{Re}(\sin z)^{2}\right) \leq-\frac{\sqrt{3}}{8}\left(e^{-2 y}+e^{2 y}\right)+\frac{1}{2}
$$

and the lemma follows. 
Lemma 4. Let $B$ be any positive constant. Then for any complex number $z$ with $|\operatorname{Re}(z)| \leq B / 4$, we have the inequality

$$
\left|\exp \left(\left(\sin \frac{z}{B}\right)^{2}\right)\right|<<\exp (-\exp (|\operatorname{Im}(z)| / B))
$$

where the constant implied by the Vinograd symbol $<<$ is absolute.

Proof. This is a corollary to Lemma 3 obtained by replacing $z$ by $z / B$.

Lemma 5. Let the maximum in (13) be attained for $\sigma=\sigma_{0} \geq 1+\delta$ and $t=t_{0}$ where $t_{0}$ is in $I_{2}$. Then the assertion of Theorem 1 holds.

Proof. Put $s_{0}=\sigma_{0}+i t$. We can certainly assume that $\sigma_{0}<2-0.01$. Let $R$ be the rectangle formed by the vertical line segments $\sigma=1, \sigma=2$ and $t$ in $I$ and the horizontal line segments connecting the upper and lower extremities of these vertical line segments. Let $D$ be the boundary of this rectangle in the anti-clockwise direction. Then by Cauchy's theorem we get

$$
\frac{1}{2 \pi i} \int_{D} \frac{\zeta(s)}{s-s_{0}} \exp \left(\left(\sin \left(s-s_{0}\right) / B\right)^{2}\right) d s=\zeta\left(s_{0}\right)
$$

Here $B$ is any positive constant. We can fix $B=4$ for our purpose. The integral along $\sigma=2, t \in I$ is $O(1)$. The integral along $\sigma=1, t \in I$ is $O\left(M \log \delta^{-1}\right)$, where $M=$ maximum of $|\zeta(1+i t)|$ as $t$ varies over $I$. The horizontal line segments $H_{1}$ and $H_{2}$ contribute

$$
O\left(\left(\exp _{2}(H / 3 B)\right)^{-1}\left(\int_{H_{1}}|\zeta(s)| d \sigma+\int_{H_{2}}|\zeta(s)| d \sigma\right)\right) .
$$

We have fixed $B=4$. Since $\zeta(s)=O(1 /(\sigma-1))$ and also $\zeta(s)=O(\log T)$, the integrals over $H_{1}$ and $H_{2}$ are

$$
O\left((\log T)(\log T)^{-1}+\int_{1+(\log T)^{-1}}^{2} \frac{d \sigma}{\sigma-1}\right)=O\left(\log _{2} T\right) .
$$

Thus

$$
\exp _{2}(H / 300)=O\left(M \log \frac{1}{\delta}+\left(\log _{2} T\right)\left(\exp _{2}(H / 12)\right)^{-1}\right) .
$$

From this and our choice of $\delta$ our assertion is proved if we make $\exp _{2}(H / 12)$ greater than $\log _{2} T$. This proves the lemma and hence Theorem 1 is completely proved. 


\section{Second application of the main theorem}

In Section 3 we saw that the proof worked because

$$
\max _{\sigma \geq 1, t \in I}|\zeta(\sigma+i t)|=O(\log T) \text { and } \max _{\sigma \geq 1, t \in I}\left|\zeta^{\prime}(\sigma+i t)\right|=O\left((\log T)^{2}\right) \text {. }
$$

By the Riemann hypothesis the corresponding estimates are $O\left(\log _{2} T\right)$ and $O\left(\left(\log _{2} T\right)^{2}\right)$. The method of proving these estimates are via $\log \zeta(s)$. An examination of the proof of these results shows that it is enough to assume that $\theta<1$. Hence we record:

Theorem 2. Let $I$ be an interval of length $H$ contained in $[T, 2 T]$. Let $T \geq H \geq C_{2} \log _{5} T$. Then

$$
\max _{t \in I}|\zeta(1+i t)| \geq e^{\gamma}\left(\log _{2} H-\log _{3} H-\varrho\right),
$$

where $\gamma$ is Euler's constant and $\varrho$ is a certain real constant.

\section{Third application of the main theorem}

Theorem 3. Let $H \leq C_{1} \log _{4} T$. Consider disjoint intervals $I$, contained in $[T, 2 T]$, all of length $H$. Put $X=\exp _{4}(\alpha H)$ where $\alpha$ is a certain positive constant satisfying $\alpha \leq C_{1}^{-1} / 2$. Then, except possibly for $O\left(T X^{-1 / 2}\right)$ intervals $I$, we have

$$
\max _{t \in I}|\zeta(1+i t)| \geq e^{\gamma}\left(\log _{2} H-\log _{3} H-\varrho\right),
$$

where $\gamma$ is Euler's constant and $\varrho$ is a certain real constant.

We prove this theorem by a few lemmas.

Lemma 1. Let $a=0.1, s=\sigma+i t$ where $T \leq t \leq 2 T$ and $1+a \geq \sigma \geq 1-a$. Then

$$
\frac{1}{2 \pi i} \int_{\operatorname{Re}(w)=2} \zeta(s+w) X^{w} \exp \left(w^{2}\right) \frac{d w}{w}=\sum_{n=1}^{\infty} \Delta\left(\frac{x}{n}\right) n^{-s},
$$

where for $u>0$ we have

$$
\Delta(u)=\frac{1}{2 \pi i} \int_{\operatorname{Re}(w)=2} u^{w} \exp \left(w^{2}\right) \frac{d w}{w} .
$$

The proof is trivial. 
Lemma 2. We have

$$
\Delta(u)=O\left(u^{5}\right) \quad \text { for } 0<u \leq 1
$$

and

$$
\Delta(u)=1+O\left(u^{-5}\right) \quad \text { for } u \geq 1 .
$$

Proof. To prove (19) we move the line of integration in (18) to $\operatorname{Re}(w)=5$ and to prove $(20)$ we move it to $\operatorname{Re}(w)=-5$.

Lemma 3. Let

$$
\zeta(s)=\sum_{n=1}^{\infty} \Delta\left(\frac{X}{n}\right) n^{-s}+E(s, X) .
$$

Then we have

$$
\int_{1-a}^{1+a} \int_{T}^{2 T}|E(s, X)|^{2} d \sigma d t=O\left(T X^{-1 / 2}\right)
$$

Proof. In the left hand side of (17) we move the line of integration to $\operatorname{Re}(w)=$ $13 / 20-\sigma$ and note that $\exp _{2}(e) \leq X \leq T$. Using

$$
\int_{T / 2}^{3 T / 2}\left|\zeta\left(\frac{13}{20}+i t\right)\right|^{2} d t=O(T)
$$

we complete the proof of the lemma.

Lemma 4. We have

$$
\sum_{n=1}^{\infty} \Delta\left(\frac{X}{n}\right) n^{-s}=\sum_{n \leq X} n^{-s}+G(s, X)
$$

where

$$
\begin{aligned}
G(s, X) & =\sum_{n \leq X}\left(\Delta\left(\frac{X}{n}\right)-1\right) n^{-s}+\sum_{n>X} \Delta\left(\frac{X}{n}\right) n^{-s} \\
& =O\left(X^{1-\sigma}\right) .
\end{aligned}
$$

Proof. Follows from Lemma 2. 
Lemma 5. The number of intervals $I$ for which

$$
\max _{1+a / 2 \geq \sigma \geq 1-a / 2, t \in I}|E(s, X)| \geq 1
$$

is

$$
O\left(T X^{-1 / 2}\right)
$$

Proof. The quantity $|E(s, X)|$ is not greater than its mean value over a disc with centre $s$ and radius $a / 2$. The lemma now follows from (22).

Lemma 6. In the region defined by $\left(1-(\log X)^{-1} \leq \sigma \leq 2, t \in I\right)$ we have

$$
\zeta(s)=O(\log X)
$$

and also in $(1 \leq \sigma \leq 2, t \in I)$

$$
\zeta^{\prime}(s)=O\left((\log X)^{2}\right)
$$

except possibly for $O\left(T X^{-1 / 2}\right)$ intervals $I$.

Proof. The equation (28) follows from Lemma 4 and 5 on noting (21). To prove (29) we may apply Cauchy's theorem to $\zeta(s)\left(s-z_{0}\right)^{-2}$ where $z_{0}$ lies in $(\sigma \geq 1, t \in I)$. We integrate over a circle with centre $z_{0}$ and radius $(\log X)^{-1}$. Rough estimates now give (29). Another proof consists in differentiating (21), (23) and (24) partially with respect to $s$. (In the second proof we have also to establish (22) with $E(s, X)$ replaced by $\partial E(s, X) / \partial s$.) Thus Lemma 6 is proved.

From Lemma 6 , Theorem 3 follows in the same way as Theorem 1 was derived in Section 3 from the estimates $\zeta(s)=O(\log t)$ and $\zeta^{\prime}(s)=O\left((\log t)^{2}\right)$ in $(\sigma \geq$ $1, t \geq 2)$. Thus Theorem 3 is completely proved, and by choosing a smaller constant $\beta$ in place of $\alpha$ our third assertion in the introduction follows.

\section{Fourth application of the main theorem}

Theorem 4. Let $H \leq C_{2} \log _{5} T$. Consider disjoint intervals $I$, contained in $[T, 2 T]$, all of length $H$. Put $Y=\exp _{5}\left(\alpha^{\prime} H\right)$ where $\alpha^{\prime}$ is a certain positive constant satisfying $\alpha^{\prime} \leq C_{2}^{-1} / 2$. Then except possibly for $O\left(T Y^{-1 / 3}\right)$ intervals $I$, we have

$$
\max _{t \in I}|\zeta(1+i t)| \geq e^{\gamma}\left(\log _{2} H-\log _{3} H-\varrho\right),
$$

where $\gamma$ is Euler's constant and $\varrho$ is a certain real constant.

We prove this theorem by a series of lemmas. Note that $\exp _{3}(e) \leq Y \leq T$. 
Lemma 1. Let $a=0.1$. The number of intervals $I$ for which

$$
\max _{1+a / 2 \geq \sigma \geq 1-a / 2, t \in I}|\zeta(s)|>>\gamma^{a / 2}
$$

is $O\left(T Y^{-1 / 2}\right)$. Here the constant implied by the Vinogradov symbol $>>$ is a certain positive constant. Let $I^{\prime}$ denote the intervals $I$ (above) with intervals of length $\log Y$ annexed at each end. Then the total length of the intervals $I^{\prime}$ is $O\left(T Y^{-1 / 2} \log Y\right)$.

Proof. The proof is similar to that of Lemmas 1 to 5 of the previous section. We have only to replace $X$ by $Y$.

Lemma 2. Let us consider the zeros $\varrho_{0}=\beta_{0}+i \gamma_{0}$ of $\zeta(s)$ with $T \leq \gamma_{0} \leq 2 T$ and $\beta_{0} \geq 1-a=0.9$. Let $\varepsilon$ be a small positive constant. With each such zero $\varrho_{0}$, we associate the rectangle $R\left(\varrho_{0}\right)$ consisting of complex numbers $z=x+i t$ satisfying $1 \geq x \geq 1-a$ and $\left|\gamma_{0}-t\right| \leq T^{\varepsilon}$. If $H\left(\varrho_{0}\right)$ denotes the height of $R\left(\varrho_{0}\right)$ then

$$
\sum_{\varrho_{0}} H\left(\varrho_{0}\right)=O\left(N(9 / 10,2 T) T^{\varepsilon}\right)
$$

where $N(9 / 10,2 T)$ denotes the number of zeros of $\zeta(s)$ with a real part $\geq 9 / 10$ and imaginary part lying between 0 and $2 T$.

The proof is trivial.

Lemma 3. From the interval $[T, 2 T]$ we omit the intervals $I^{\prime}$ of Lemma 1 of total length $O\left(T Y^{-1 / 2} \log Y\right)$ and also the $t$-intervals counted in Lemma 2 of total length $O\left(N(9 / 10,2 T) T^{\varepsilon}\right)$. Then the maximum number of intervals $I$ which have at least one point in common with these $t$-intervals is

$$
O\left(T Y^{-1 / 2} H^{-1} \log Y\right)+O\left(N(9 / 10,2 T) H^{-1} T^{\varepsilon}\right) .
$$

Proof. We have only to annex on either side of the excluded intervals $t$ intervals of length $H$. We then exclude the maximum possible number of $t$ intervals $I$ which are wholly contained in the union of extended intervals.

Lemma 4. We have

$$
N(9 / 10,2 T)=O\left(T^{1 / 3}(\log T)^{50000}\right)
$$

and so the maximum possible number of intervals $I$ (which are excluded) is

$$
O\left(T Y^{-1 / 2} H^{-1} \log Y\right) \text {. }
$$

Proof. Using only the mean square result regarding $|\zeta(1 / 2+i t)|$ we can prove the result $N(\sigma, T)=O\left(T^{\lambda(1-\sigma)}(\log T)^{50000}\right)$ where $\lambda=4 /(3-2 \sigma)$. (See [6] and the references therein.) This is the simplest non-trivial density result.

(The method of obtaining such results can be traced to many authors. See [11] and [4].) This result gives the lemma. We can choose $\varepsilon=1 / 4$. 
Lemma 5. Let $I$ be a $t$-interval which is not excluded by Lemmas 3 and 4. (We will prove the expected lower bound for the maximum of $|\zeta(1+i t)|$ taken over such intervals.) Then for any point $t_{1}$ belonging to $I$, the rectangle $S\left(t_{1}\right)$ defined by

$$
S\left(t_{1}\right)=\left\{\sigma+i t|0.9 \leq \sigma \leq 2,| t-t_{1} \mid \leq \log Y\right\}
$$

is free from zeros of $\zeta(s)$ and also $|\zeta(s)| \leq Y$ there.

Proof. Follows from Lemmas 1 to 4.

Lemma 6. Let $C_{6}$ be a large positive constant. Then in the rectangle $U\left(t_{1}\right)$ defined by

$$
U\left(t_{1}\right)=\left\{\sigma+i t|0.95 \leq \sigma \leq 2,| t-t_{1} \mid \leq \log Y-C_{6}\right\}
$$

we have $\log \zeta(s)=O(\log Y)$.

Proof. The lemma follows by a suitable application of the Borel-Caratheodory theorem (see p. 282 of [11]).

Lemma 7. Let $s$ by any point of the rectangle $V\left(t_{1}\right)$ defined by

$$
V\left(t_{1}\right)=\left\{\sigma+i t|0.975 \leq \sigma \leq 2,| t-t_{1} \mid \leq \frac{1}{2} \log Y-C_{6}\right\} .
$$

Let $V=(\log Y)^{100}$ and let

$$
\log \zeta(w)=\sum_{n=2}^{\infty} a_{n} n^{-w}
$$

in $\operatorname{Re}(w) \geq 2$. Then for $s$ in $V\left(t_{1}\right)$, we have

$$
\frac{1}{2 \pi i} \int_{\operatorname{Re}(w)=2} \log \zeta(s+w) V^{w} \exp \left(w^{2}\right) \frac{d w}{w}=\sum_{n=2}^{\infty} \Delta\left(\frac{V}{n}\right) a_{n} n^{-s},
$$

where $\Delta(u)$ for $u>0$ is defined as in (18).

The proof is trivial.

Lemma 8. We have uniformly for all $s$ in $V\left(t_{1}\right)$

$$
\sum_{n=2}^{\infty} \Delta\left(\frac{V}{n}\right) a_{n} n^{-s}=\log \zeta(s)+o(1) .
$$

Proof. Let us consider the integral in (39). The contribution from $|\operatorname{Im}(w)| \geq$ $(\log Y) / 4$ is $o(1)$ since $\left|\exp \left(w^{2}\right)\right|<<\exp \left(-|\operatorname{Im}(w)|^{2}\right)$ where the constant implied by the Vinogradov symbol is absolute if $|\operatorname{Re}(w)|$ does not exceed an absolute constant. We deform the rest of the contour as follows. $\operatorname{Im}(w)=-(\log Y) / 4$ in the direction of $\operatorname{Re}(w)$ decreasing from 2 to $0.95-\sigma$; then the vertical line $\operatorname{Re}(w)=0.95-\sigma$ in the direction of $\operatorname{Im}(w)$ increasing and then $\operatorname{Im}(w)=(\log Y) / 4$ in the direction of $\operatorname{Re}(w)$ increasing from $0.95-\sigma$ to 2 . Using Lemma 6 it is easily seen that the integrals along the deformed contour contribute $o(1)$. The pole $w=0$ contributes $\log \zeta(s)$. Thus the lemma is completely proved. 
Lemma 9. For $s$ in $V^{*}\left(t_{1}\right)$, defined by

$$
V^{*}\left(t_{1}\right)=\left\{\sigma+i t\left|1-(\log V)^{-1} \leq \sigma \leq 2,\right| t-t_{1} \mid \leq \frac{1}{2} \log Y-C_{6}\right\},
$$

we have the estimates $\zeta(s)=O(\log V)$ and, in the part $\sigma \geq 1$ of $V^{*}\left(t_{1}\right), \zeta^{\prime}(s)=$ $O\left((\log V)^{2}\right)$ with $V=(\log Y)^{100}$.

Proof. Let $\sigma^{*}=1-(\log V)^{-1}$. Then in the region $V^{*}\left(t_{1}\right)$ we have

$$
\begin{aligned}
|\log \zeta(s)| & \leq \sum_{n=2}^{\infty} \Delta\left(\frac{V}{n}\right) a_{n} n^{-\sigma^{*}}+O(1) \leq \sum_{p} \Delta\left(\frac{V}{p}\right) p^{-\sigma^{*}}+O(1) \\
& \leq \sum_{p \leq V} p^{-\sigma^{*}}+O(1) \leq \sum_{p \leq V} p^{-1}+O(1) \leq \log _{2} V+O(1) .
\end{aligned}
$$

Here the first three inequalities follow as in Lemma 4 of the previous section. The fourth follows since for $p \leq V, p^{-\sigma^{*}}=p^{-1}+O\left(p^{-1} \log p / \log V\right)$ and since $\sum_{p \leq V} \log p / p=O(\log V)$ by prime number theorem. The last inequality follows by prime number theorem. The first estimate of the lemma follows since $\log |\zeta(s)| \leq|\log \zeta(s)|$. The second follows from the first by applying Cauchy's theorem. Thus Lemma 9 is completely proved.

Theorem 4 follows from Lemma 9 (just as we derived Theorem 1 from the main theorem). Our fourth assertion in the introduction follows from Theorem 4.

\section{Concluding remarks}

The arguments of the previous section resemble to some extent the definition and treatment of the Huxley-Hooley contour in [5]. We can work out the results corresponding to the previous sections for $1 / 2<\sigma<1$ and also for $\sigma=1 / 2$. Thus

$$
\max _{t \in I}|\zeta(\sigma+i t)|>\exp \left(\frac{C_{7}(\log H)^{1-\sigma}}{\log _{2} H}\right)
$$

holds with the exception of at most $O\left(T\left(\exp _{2}\left(\beta^{\prime \prime} H\right)\right)^{-1}\right)$ intervals where $\beta^{\prime \prime}$ is a positive constant, provided $C_{4} \leq H \leq C_{8} \log _{2} T$ ( $C_{8}$ being any positive constant and $\beta^{\prime \prime}$ is allowed to depend on $C_{8}$ ).

On $\sigma=1 / 2$ we get

$$
\max _{t \in I}\left|\zeta\left(\frac{1}{2}+i t\right)\right|>\exp \left(\frac{3}{4}\left(\frac{\log H}{\log _{2} H}\right)^{1 / 2}\right)
$$

(some positive constant in place of $3 / 4$ comes out by [2], but $3 / 4$ comes out by using a result in [1]) with exceptions nearly the same as before but with an extra restriction $H \geq \log _{3} T$.

The improvement of these results seems to be difficult. (For some kernel functions used in the present paper see [9] and the reference list there, especially the reference number 3.) 


\section{References}

[1] Balasubramanian, R.: On the frequency of Titchmarsh's phenomenon for $\zeta(s)-$ IV. Hardy-Ramanujan J. 9, 1986,1-10.

[2] Balasubramanian, R., and K. Ramachandra: On the frequency of Titchmarsh's phenomenon for $\zeta(s)$-III. - Proc. Indian Acad. Sci. Math. Sci. 86, 1977, 341-351.

[3] Balasubramanian, R., and K. Ramachandra: Progress towards a conjecture on the mean-value of Titchmarsh series, III. - Acta Arith. XLV, 1986, 309-318.

[4] IvIC, A.: The Riemann zeta-function. - John Wiley and Sons, New York-ChichesterBrisbane-Toronto-Singapore, 1985.

[5] Ramachandra, K.: Some problems of analytic number theory, I. - Acta Arith. XXXI, 1976, 313-324.

[6] Ramachandra, K.: Riemann zeta-function. - Publications of the Ramanujan Institute 4, University of Madras, 1979.

[7] Ramachandra, K.: Progress towards a conjecture on the mean-value of Titchmarsh series, I. - Recent progress in analytic number theory (edited by H. Halberstam and C. Hooley), I. Academic Press, London-New York-Toronto-Sydney-San Francisco, 1981, 303-318.

[8] RamachandRa, K.: A brief summary of some results in the analytic theory of numbers, II. - Addendum. Number theory, Proceedings of a conference held in Mysore in 1981 (edited by K. Alladi). Lecture Notes in Mathematics 938. Springer-Verlag, BerlinHeidelberg-New York, 1982, 106-122.

[9] Ramachandra, K.: Mean-value of the Riemann zeta-function and other remarks, III. Hardy-Ramanujan J. 6, 1983, 1-21.

[10] Ramachandra, K., and A. Sankaranarayanan: Omega-theorems for the Hurwitz zeta-function. - Ark. Mat. (to appear).

[11] Titchmarsh. E.C.: The theory of the Riemann zeta-function. - Clarendon Press, Oxford, 1951.

Tata Institute of Fundamental Research

School of Mathematics

Homi Bhabha Road

Bombay 400005

India

Received 6 October 1987 